\title{
Adaptive Beamforming and Power Allocation for OFDM Over Wireless Networks
}

\author{
Masoud Olfat, K. J. Ray Liu \\ Electrical Engineering Department \\ University of Maryland \\ College Park, MD 20742 \\ (molfat, kjrliu@isr.umd.edu)
}

\author{
Farrokh. Rashid-Farrokhi \\ Wireless Communications Research Dept. \\ Bell Labs, Lucent Technologies \\ 791 Holmdel-Keyport Rd, Rm. R-111 \\ Holmdel, NJ 07733
}

\begin{abstract}
In this paper, the performance of a multiuser wireless network using OFDM, combined with Power Control and Adaptive Beamforming for uplink transmission is presented. An adaptive power control algorithm is exploited to acheive the desired Signal to Noise and Interference Ratio(SINR) at each subchannel and increase the power efficeincy of the mobile transmitter. In the receiver side, the base station uses an antenna array to optimize the SNR-Power effceincy, and attenuate the interference from other users dramatically. Therefore, we can achieve a better overall error probability with a fixed total power. A distributed iterative algorithm is used to jointly update the transmission power and the beamformer weights at each subchannel so that it can converge to the optimal solution for both power and beamforming vectors at each subchannel. The algorithm uses only the interference measured locally by the transmitter. Unlike most of the loading algorithms which optimize the bit distribution and subchannel power allocation for a single transmitter, this approach tries to optimize the power allocatoin and decrease the interference for the whole network.
\end{abstract}

\section{Introduction}

Orthogonal Frequency Division Modulation(OFDM) is a parallel data transmision scheme. OFDM systems employ several techniques such as: frequency and/or time interleaving, time gaurd band, different coding strategies, simple equalization.

OFDM has several advantages and disadvantages. Among its advantages, high bandwidth efficeincy, converting a wideband frequency selective fading channel into a series of narrowband flat fading subchannels, averaging the time domain short term distortion, due to FFT operation at the receiver side, and relatively large block duration, no need for sophisticated equalization method, are considerable.

One problem with OFDM is its poor performance due to the sensitivity of error probability to the subcarrier with the lowest signal to noise ratio. Also, the error probability decreases slowly with increasing signal power [1].

Several methods have been proposed to combat the aforementioned problem. Those methods are basically trying to adjust the bit and power distribution among subchannels according to their performances and are mostly called "loading algorithms" [2], [3], [5] and [8]. However, most of them have considered a single transmitter. In a mobile environment, each user's signal can affect others and this, in turn results in further attenuation.

Power control is an appropriate solution to keep the SINR of all subcarriers in a desired level and minimize the interference caused by other users as much as possible, and to achieve better bit error rate. In the receiver side, An adaptive beamformer, further attenuates the intereference caused by other users.

This paper is organized as follows: Section 2 introduces the basics of OFDM systems, the problem of power control and its solution. Section 3 along with Section 4 introduces our proposed system and some simulation results and Section 5 concludes the paper.

\section{Basic concepts}

\subsection{Orthogonal Frequency Division Multiplexing}

Fig.1 shows the basic building block of an OFDM transmitter and receiver. Consider a data sequence $s_{0}, s_{1}, s_{2}, \ldots$, where each $s_{n}$ represents a symbol of $m_{n}$ bits. Everey $N$ symbols are combined to make a block of data. Each symbol passes through a modulator, which can be QPSK or QAM, resulting a sequence of complex numbers 
$d_{0}, d_{1}, d_{2}, \ldots d_{N-1}$.

If an Inverse Discrete Fourier Transform(IDFT) operation is performed on this block, the result is another vector of $N$ complex numbers $X_{0}, X_{1}, \ldots, X_{N-1}$.

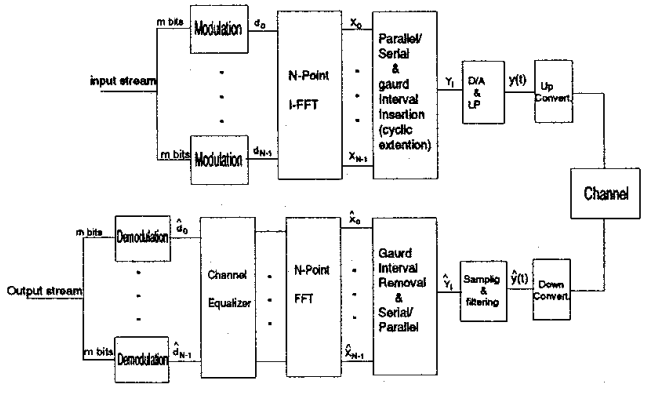

Figure 1. Basic Building blocks of an OFDM transmitter and reciever.

The real and imaginary parts of the modulated symbols are separated and the parallel data are converted back to serial. By applying these components to a low pass filter at time intervals $T_{s}$ (block length) the inphase and the quadrature components of OFDM signal are obtained. These components are up-converted in order to be transmitted through the channel.

At the receiver, the received signal is down converted to baseband and the inphase and quadrature components of the OFDM signal are extracted.

Assuming perfect block synchronization between the transmitter and the receiver, the latter is able to extract the relevant symbol interval $T_{s}$, and sample the components of received signal at the multiples of $T_{s}$ to obtain the complex samples $\hat{X}_{k}(k=0, \ldots, N-1)$. These samples are used to perform a DFT operation and the resultant symbols are being demodulated to estimate symbols $\hat{s}_{n}$.

The transmitter introduces a cyclic extension gaurd interval $T_{G}$ (larger than the delay spread of the channel) before the low pass filter to preserve the orthogonality of the subchannels at the presence of InterSymbol Interference(ISI) caused by channel distortion and multipath delays. So, if the receiver neglects the received signal outside the time interval $T_{s}$, the effect of ISI is avoided.

As was mentioned in previous section, several loading algorithms have been proposed to adjust the rate and power at each subchannel. For example Chow and Coiff [2] proposed a method in which they distribute the bits according to the capacity of the subchannels. They have used the concept of "SNR gap approximation $\Gamma$ ", where $10 \log \Gamma$ is the SNR gap between the subchannel capacity and the bandwidth efficiency of the real modulation block. In each iteration, they use the following formula to find the number of bits assigned to each subchannel (after rounding to the maximium integer)

$$
R_{n}=\log _{2}\left(1+\frac{\gamma_{n}}{\Gamma+\gamma_{m}}\right)
$$

when $\gamma_{n}$ is the desired SNR of the subchannel $n$ and $\gamma_{m}$ is the difference between the actual and desired SNR of the subchannel. The criteria here, is to minimize the overall error probability.

Fisher [5] exploits the fact that the signal power and the rate at each subchannel are related. He tries to fix the data rate and transmitted signal power is each subchannel and transmit at the lowest possible error rate. He charactrizes the subchannels as AWGN and use $p_{e}(i)=K_{i} Q\left(\sqrt{\frac{d_{i} / 4}{N_{i} / 2}}\right)$ to find the symbol arror rate at each subchannel (assuming QAM), where $Q($.$) is the Gaussian pdf tail, d_{i}$ is the minimum Euclidian distance betwen signal points in the constellation and $N_{i} / 2$ is the noise power.

\subsection{Power Control}

The objective of power control in wireless netwroks is to minimize the transmitted power when at the same time the target error probabilities are met. To do this, we should keep each mobile's Signal to Interference and Noise Ratio (SINR) above a threshold called minimum protection ratio. We denote the link gain between the $j$ th mobile and $i$ th base station by $G_{j i}$, and the $j$ th mobile transmitted power by $P_{j}$. Note that, $G_{j i}$ encompasses the effect of shadow fading, attenuation due to distance and the frequency shift. The SINR at the $i$ th receiver is given by

$$
\Gamma_{i}=\frac{G_{i i} P_{i}}{\sum_{j \neq i} G_{j i} P_{j}+N_{i}},
$$

where $N_{i}$ is the thermal noise at the $i$ th base station. Our objective is to maintain the transmitted power as low as possible and at the same time keep the SINRs above the threshold. Using $\Gamma_{i}=\gamma_{i}$ and the Perron- Frobenius theorem[6], the $i$ th mobile power is updated by

$$
P_{i}^{n+1}=\frac{\gamma_{i}}{G_{i i}}\left(\sum_{j \neq i} G_{j i} P_{j}^{n}+N_{i}\right) \quad i=1, \ldots, M
$$

The right hand side in (3) is a function of the interference at the $i$ th mobile (the quantity inside the paranthesis), the link gain $G_{i i}$, and the target SINR. All of these parameters can be measured locally by the base station and transmitted through a feedback channel to the correspondant mobile.

\section{The Proposed System}

As we mentioned earlier, in a multicarrier system, the error probability of the whole system is affected significantly 
by the subchannel with the highest attenuation. Therefore in the case of frequency selective fading channel, the performance of the whole system in terms of error probability will improve slowly by increasing the transmitted power. So, in order to get a minimum overall error probability, the optimum procedure, in a fixed total power policy, is to have a uniform error probability for all of the subchannels [1].

However, in a cellular environment, there are cochannel interferences between different mobiles trying to use the same subchannel. In this situatuion the amount of interference at each subchannel for each mobile is directly related to the power of other mobiles using the same subchannel, and so is the SINR. Therefore, the individual loading algorithms do not come up with the optimum power and bit distribution. In this paper, our objective is to optimize the power distribution at each subchannel for all of the mobiles, so that

1-The SINR is fixed at all of the subchannels for all of the mobiles, and therefore the error probability decreases faster with growing SINR compared with that of unbalanced SINR.

2-The total power used to achieve the aforementioned objective is minimized.

The basic idea behind this, is to allocate less power to the subchannels with better performances, and more power to the subchannels with low SINR.

Our assumptions here are, the same modulation scheme at all of the subchannels (different SINRs) and no interchannel interferences (independent subchannels). With these, we perform the power control algorithm mentioned in previous section for each subchannel separately.

Fig. 2 depicts the proposed system for the mobiles using OFDM scheme. In the first case, we assume a simple OFDM receiver at the base stations as in Fig. 1.

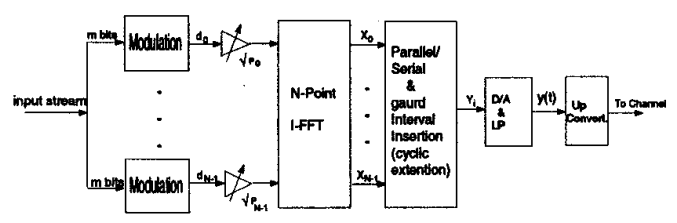

Figure 2. OFDM Transmitter using adaptive power allocation.

By applying the adaptive power control algorithm, we gaurantee that the ratio of the desired signal power to the combination of interference and noise at $l$ th subchannel is at least a prespecified value $\gamma_{l}$.

In Fig. 3 (second case), we have considered antenna diversity for the receiver. Multiple antenna elements have been used at each receiver. The joint beamforming and power control procedure is performed at each subchannel separately, assuming perfect orthogonalization.

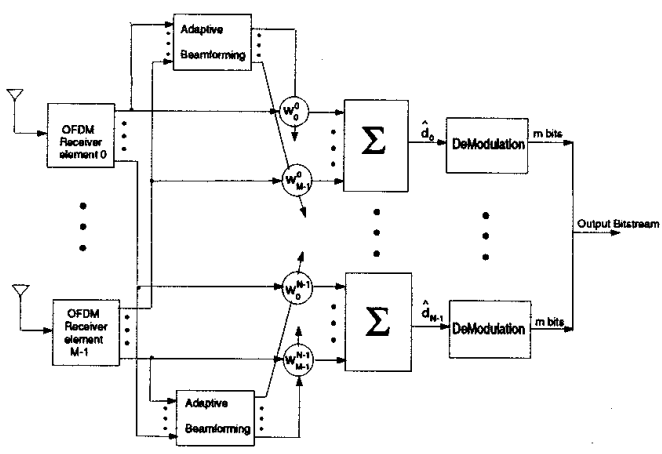

Figure 3. Antenna Array in an OFDM Receiver

The adaptive beamforming vector is calculated to minimize the total energy, when the gain toward the desired signal is constant.

We denote the power allocated to mobile $i$ at subchannel $k$ as $P_{i}^{k}$, a sample of a white gaussian noise at subchannel $k$ by $n^{k}$ and its Fourier transform by $\hat{n}^{k}$ and the array response for the signal coming from mobile $i$ to base $j$ by $\mathbf{a}_{i j}$.

The received vector at the $j$ th base station and $k$ th subchannel(assuming negligible delay spread from different paths) is given by

$$
\mathbf{x}_{j}^{k}=\sum_{i=0}^{M-1}\left(\sum_{l=0}^{N-1} \sqrt{P_{i}^{l} G_{i j}^{l}} d_{i}^{l} e^{-j 2 \pi l k / N}\right) \mathbf{a}_{i j}+\mathbf{n}^{k} .
$$

At the input of each antenna, the input samples are used to perform Fourier Transformation. The resultant $l$ th output of the FFT block at each antenna is the following vector

$$
\hat{\mathbf{d}}_{j}^{l}=\sum_{i=0}^{M-1} \sqrt{P_{i}^{l} G_{i j}^{l}} d_{i}^{l} \mathbf{a}_{i j}+\hat{\mathbf{n}}^{l} . \quad l=0, \ldots, N-1
$$

Assuming that the noise samples are zero mean, independent gaussian variables, and the transmitted symbols are independent and have average energy equal to 1 , the output energy will be

$$
\epsilon^{l}=\mathbf{w}_{j}^{l}{ }^{H} E\left[\hat{\mathbf{d}}_{j}^{l} \hat{\mathbf{d}}_{j}^{l}{ }^{H}\right] \mathbf{w}_{j}^{l}
$$

$=\left[\sum_{i \neq j_{i=0}}^{M-1}(P_{i}^{l} G_{i j}^{l} \underbrace{\mathbf{w}_{j}^{l}{ }^{H} \mathbf{a}_{i j} \mathbf{a}_{i j}^{H} \mathbf{w}_{j}^{l}}_{u_{i j}^{l}})+\frac{N_{0}}{2}\left\|\mathbf{w}_{j}^{l}\right\|^{2}\right]+P_{j}^{l} G_{j j}^{l} u_{j j}^{l}$,

in which the term inside the bracket is the interference plus noise and the second term is the power of the signal coming from desired direction. 
The signal to noise ratio at the output of the beamformer is given by

$$
\Gamma_{j}^{l}=\frac{P_{j}^{l} G_{j j}^{l} u_{i j}^{l}}{\sum_{i \neq j_{i=0}}^{M-1}\left(P_{i}^{l} G_{i j}^{l} u_{i j}^{l}\right)+\frac{N_{0}}{2}\left\|\mathbf{w}_{j}^{l}\right\|^{2}} .
$$

The problem of beamforming will be

$$
\begin{aligned}
\mathbf{w}_{j}^{l}= & \left.\underset{\mathbf{w}}{\arg \min _{i=0_{i \neq j}}}\left\{\sum_{i}^{M-1}\left(P_{i}^{l} G_{i j}^{l} u_{i j}^{l}\right)+\frac{N_{0}}{2}\left\|\mathbf{w}_{j}^{l}\right\|^{2}\right]\right\} \\
& \text { subject to } \mathbf{w}_{j}^{l} \mathbf{a}_{i j}=1
\end{aligned}
$$

Using a lagrange multiplier [7] the optimum weight vectors are obtained by

$$
\mathbf{w}_{j}^{l}=\frac{\mathbf{R}_{j}^{l^{-1}} \mathbf{a}_{i j}}{\mathbf{a}_{i j}{ }^{H} \mathbf{R}_{j}^{l-1} \mathbf{a}_{i j}} . l=0, \ldots, N-1
$$

when

$$
\mathbf{R}_{j}^{l}=\sum_{i=0}^{M-1}\left(P_{i}^{l} G_{i j}^{l} \mathbf{a}_{i j} \mathbf{a}_{i j}^{H}\right)+\frac{N_{0}}{2} \mathbf{I}
$$

is the correlation matrix at subchannel $l$ at base station $j$.

The following algorithm achieves the jointly optimal power allocations and adaptive beamforming at each subchannel:

1-At subchannel $l$, using (8) the wieght vector $\mathrm{w}_{j}^{l}(n+1)$ is computed at each receiver $j$ such that the cochannel interference is minimized. To evaluate the correlation matrix, we use $\mathbf{P}_{j}^{l}(n)$ and $\mathbf{w}_{j}^{l}(n)$, the power vector and the weight vector updated at step $n$, in place of $P_{i}^{l}$ and $\mathbf{w}_{j}^{l}$. by

2-The updated power vector, $P_{j}^{l}(n+1)$, is then obtained

$P_{j}^{l}(n+1)=\frac{\gamma_{l}}{G_{j j}^{l}}\left[\sum_{i \neq j_{i=0}}^{M-1}\left(G_{i j}^{l} P_{j}^{l}(n) u_{i j}^{l}(n)\right)+\frac{N_{0}}{2}\left\|\mathbf{w}_{j}^{l}\right\|^{2}(n)\right]$

By looking at (8) and a simple calcuation, we can see that if $\alpha$ is the denominator of $(8), P_{j}^{l}(n+1)$ can be evaluated by $P_{j}^{l}(n+1)=\frac{\Gamma_{l}}{\alpha G_{j j}^{l}}$, too.

It has been shown that the above algorithm converges to the optimal power allocation and beamforming vectors and the solution is unique [4].

\section{Simulation Results}

we have used a 36 base stations wireless network (one mobile for each), 64 subchannels QPSK OFDM systems, a gaussian white noise with $1 \mathrm{MHz}$ noise bandwidth

\begin{tabular}{ccccc}
\hline System setup & antenna & power & policy & SINR \\
\hline 1 & single & adaptive & - & fixed \\
2 & single & uniform & - & variable \\
3 & multiple & adaptive & joint & fixed \\
4 & multiple & uniform & - & variable \\
5 & multiple & adaptive & tandem & fixed
\end{tabular}

Table 1. simulation Ssytem setups

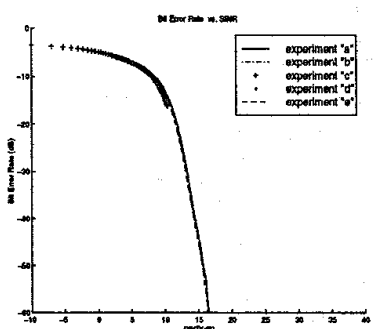

(a)

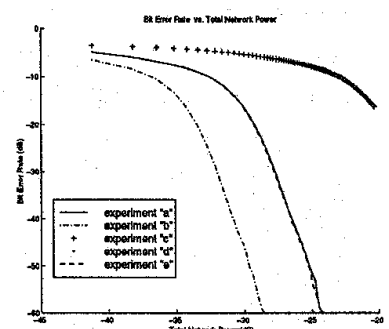

(b)
Figure 4. Fixed Total Power for single antenna cases (a) The bit error rate $(\mathrm{dB})$ versus desired $\operatorname{SINR}(\mathrm{dB})$. (b)The bit error rate $(\mathrm{dB})$ versus total network power $(\mathrm{dB})$.

( $4 X 10^{-15}$ variance) and a range of $0 \mathrm{~dB}$ to $20 \mathrm{~dB}$ for SINR and $12 \mathrm{Mbit}$ data file. Several experiments have been performed for each value of SINR. Different path gains are used for different mobiles and also for different subchannels to reflect the effect of frequency selective fading channels, but at each subchannel, flat fading has been considered.

Table 1 shows the system setups for different experiments. In experiment ' $a$ ' we have used setup 1 and a typical base station. A typical base station, the worst, the best, and the average base station (in terms of SINR) along with system setup 2 are used in experiments ' $b{ }^{\prime},{ }^{\prime} c{ }^{\prime},{ }^{\prime} d^{\prime}$ and ' $e^{\prime}$, respectively, in which the same total network as experiment ' $e$ ' is divided uniformly between all of the mobiles and all of the subchannels. Experiments ${ }^{\prime} h^{\prime},{ }^{\prime} i^{\prime}$ and ${ }^{\prime} j^{\prime}$ use a typical base station along with setup 3,4 and 5 respectively.

As it is observable from Fig. (4.a), in the single antenna case, the bit error rate versus SINR follows the same pattern. The reason is that we are using the same modulation scheme for all of the subchannels and all of the mobiles. Knowing the SINR, the bit error rate is well-known for a particular modulaion scheme.

Fig. (4.b) shows that using a fixed power policy, some base stations can achieve much better bit error rate than case $a$. At the same time, compared to the adaptive power allocation scheme, the performance of some other cases is much lower. The point is, by power allocation we specify a lower bound for SINR at all of the subchannels and 


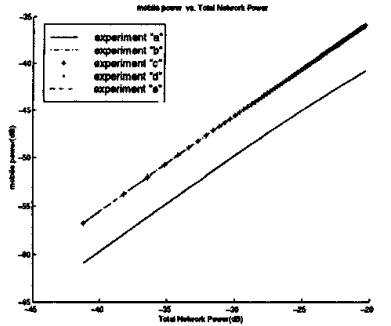

(a)

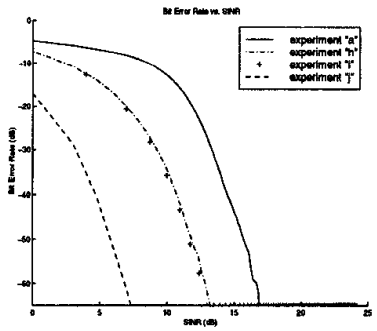

(b)
Figure 5. (a) The mobile power $(\mathrm{dB})$ versus Total power network (dB) for system setup 1(b) The bit error rate(dB) versus SINR(dB) comparing system setups.

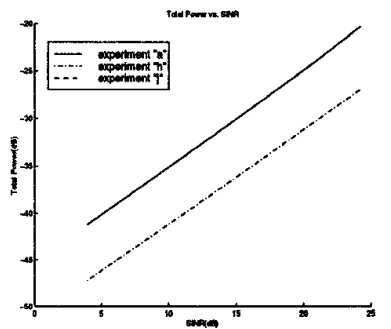

(a)

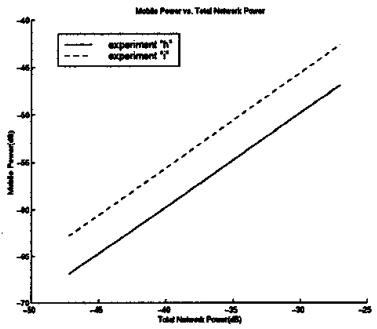

(b)
Figure 6. (a) The Total Power(dB) versus SINR(dB) comparing system setups (b) Mobile power(dB) versus total network power(dB) comparing setup 3 and 4

so all of the subchannels at all of the mobiles have SINR in the vicinity of the desired value to minimize the total power. If we divide this power uniformly, it is natural that different subchannels at different mobiles, based on their channel responses, perform differently. By power control, all of the subchannels perform at the same level of performance. Also, it is clear from Fig. (5.a) that those base stations which perform better in terms of bit error rate in a fixed power policy, consume more power than the adaptive policy and so the overall performance is depreciated.

Fig. (5.b) shows the bit error rate versus SINR for the cases using multiple antenna elements at the receiver. The joint policy performs better than single antenna, but lower than the tandem policy, but Fig. 6.a shows that this is achieved with the cost of much higher total power for the network. So the overall performance of the joint policy, in terms of total network power versus a prespecified SINR is optimized.

Fig. (6.b) compares the mobile power for cases ' $h$ ' and
$' i{ }^{\prime}$. Having the same total network power, the mobile power is lower in adaptive power allocation scheme.

Through simulation, It was observed that when SINR reaches to $19 \mathrm{~dB}$, no fixed power allocation is feasible. The joint power allocation and beamforming gaurantess a level of performance for the whole network with a certain total power.

The joint power control and beamforming scheme converges faster than the single antenna case, in terms of number of mobiles and the distance between the mobile and the base station (base dimension). In other words the frequency reuse at each subchannel has increased signifacantly.

\section{Conclusion}

An iterative and adaptive joint power control and beamforming algorithm proposed in [4] has been used to fix the signal to noise and interference ratio and minimize the cochannel interference at each subchannel of an OFDM system in a network of mobiles. We have used the total mobile power and the total transmitted power in the whole network as another measures of performance. The proposed system tries to optimize the tradeoff between the transmitted power and bit error rate for the whole network, not for a single transmitter. We have shown when we use adaptive antenna at the base station using an OFDM system, the effects of interference and white noise diminishes more significantly. This also speeds up the convergence of the iterative algorithm.

\section{References}

[1] J. A. C. Bingham. Multicarrier modulation for data transmission: An idea whose time has come. Communication Magazine, pages 5-14, May 1990.

[2] J. A. C. Bingham, J. M. Coiffi, and P. S. Chow. A practical discrete multitone tranceiver loading algorithm for data transmission over spectrally shaped channels. IEEE Trans. On Communications, February 1995.

[3] A. Czylwik. Adaptive ofdm for wideband radio channels. IEEE Globecom, 1996.

[4] F. R. Farrokhi, L. Tassiulas, and K. J. R. Liu. Joint optimal power control and beamforming in wireless networks using antenna arrays. IEEE Trans. on Communications, 46(10):1313-1324, October 1998.

[5] R. F. H. Fisher and J. B. Huber. A new loading algorithm for discrete multitone transmission. Proc. Of GlobalCOM, February 1996.

[6] F. R. Gantmacher. The Theory of Matrices. Chelsea, New York, third edition, 1990.

[7] S. Haykin. Adaptive Filter theory. Prentice Hall, third edition, 1996.

[8] D. Hughes and Hartogs. Ensembled modem structure for imperfect transmission media. U.S. Patent Notes 4679226, July 1987. 